\title{
Optically interrogated MEMS pressure sensor array
}

\section{Journal Article}

Author(s):

Prochazka, Lukas; Meier, Alexander H.; Viggiani, Antonio; Rösgen, Thomas

Publication date:

2012-04

Permanent link:

https://doi.org/10.3929/ethz-b-000041613

Rights / license:

In Copyright - Non-Commercial Use Permitted

Originally published in:

Experiments in Fluids 52(4), https://doi.org/10.1007/s00348-011-1182-3 


\title{
Optically interrogated MEMS pressure sensor array
}

\author{
Lukas Prochazka • Alexander H. Meier • \\ Antonio Viggiani · Thomas Roesgen
}

Received: 19 October 2010/Revised: 14 June 2011/Accepted: 1 August 2011/Published online: 23 August 2011

(C) Springer-Verlag 2011

\begin{abstract}
A novel pressure measurement technique is presented for wireless recording of time-averaged surface pressure distributions in wind tunnel surveys. An array of silicon micro-plate resonators acts as pressure sensing element. The pressure is recorded by measuring the sensor diaphragms' resonance frequency using optical interferometry. Dependent on the quasi-static deflection caused by a pressure load, the resonance frequency varies with an average pressure sensitivity of $3 \mathrm{~Hz} / \mathrm{Pa}$ in a frequency range between 30 and $150 \mathrm{kHz}$. A smart-pixel CMOS camera, narrow-band acoustic noise excitation and a specific sensor surface structure allow for the interrogation of a large number of sensors in parallel without the need for alignment between sensor and detector. Experimental tests reveal increased sensing performance with acoustic excitation of the higher vibration modes.
\end{abstract}

\section{Introduction}

During aerodynamic wind tunnel testing, it is often useful to record the surface pressure distribution on the test article. Such measurements require high spatial resolution, good accuracy, and low uncertainty. In industrial applications, the pressure tap method is most often used to measure the static

L. Prochazka $(\bowtie) \cdot$ A. H. Meier · A. Viggiani · T. Roesgen Institute of Fluid Dynamics, ETH Zurich, Sonneggstrasse 3, 8092 Zurich, Switzerland

e-mail: prochazka@ifd.mavt.ethz.ch

A. H. Meier

e-mail: meier@ifd.mavt.ethz.ch

A. Viggiani

e-mail: vtony@student.ethz.ch

T. Roesgen

e-mail: roesgen@ifd.mavt.ethz.ch pressure. Here, each pressure tap forms a small orifice on the surface of the model, while being linked to the pressure transducer through a pneumatic tube. A multi-tap system provides a good measurement performance, but is also very costly because of the mechanical and pneumatic interfaces to the test model. Furthermore, the integration into thin structures such as flaps can be difficult or even infeasible. Another technique, the application of pressure sensitive paints for lowspeed testing at atmospheric pressure conditions is often inaccurate, suffering from inherently low pressure sensitivity and high cross-response to temperature variations (Airaghi 2006; Liu et al. 2005).

In the present work, a new pressure sensing technique will be introduced with a performance comparable to that of a pressure tap system, but with reduced installation and handling complexity. The two most important components of the new system are the surface-mounted passive MEMS pressure sensor dies and a highly sensitive active-pixel CMOS lock-in detector, enabling wireless interrogation of many sensors in parallel. Typical performance requirements are summarized in Table 1.

In the following, the relevant aspects of the measurement system will be described, in particular, the sensor fabrication and calibration. The sensing performance is evaluated both in a dedicated test cell and in a representative wind tunnel environment. A single-point laser Doppler vibrometer is used as the reference sensing instrument. The feasibility of fullfield interrogation using an imaging interferometer is then demonstrated using a demodulating smart-pixel camera.

\section{Sensing principle}

The sensing principle is based on the optical interrogation of an array of surface-mounted MEMS pressure sensors 
Table 1 Required measurement performance

\begin{tabular}{lc}
\hline Temporal resolution & $\approx 1 \mathrm{~Hz}$ \\
Spatial resolution & $>5 \mathrm{~mm}$ \\
Measurement range & -9 to $3 \mathrm{kPa}$ (signed \\
& pressure measurement) \\
High accuracy (high sensitivity, & $0.2 \% \mathrm{FS}$ or $20 \mathrm{~Pa}$ \\
$\quad$ low measurement uncertainty) & \\
\hline
\end{tabular}

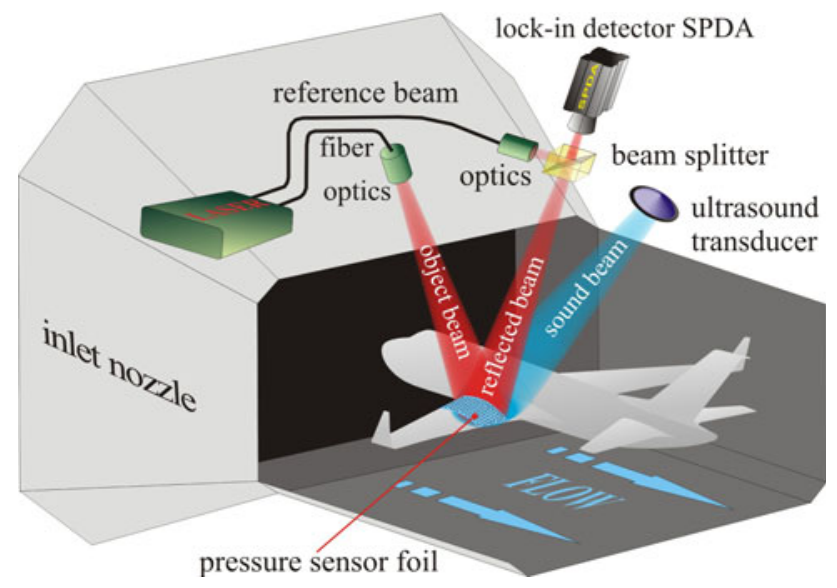

Fig. 1 Measurement setup including the remote imaging interferometer and the ultrasound transducer

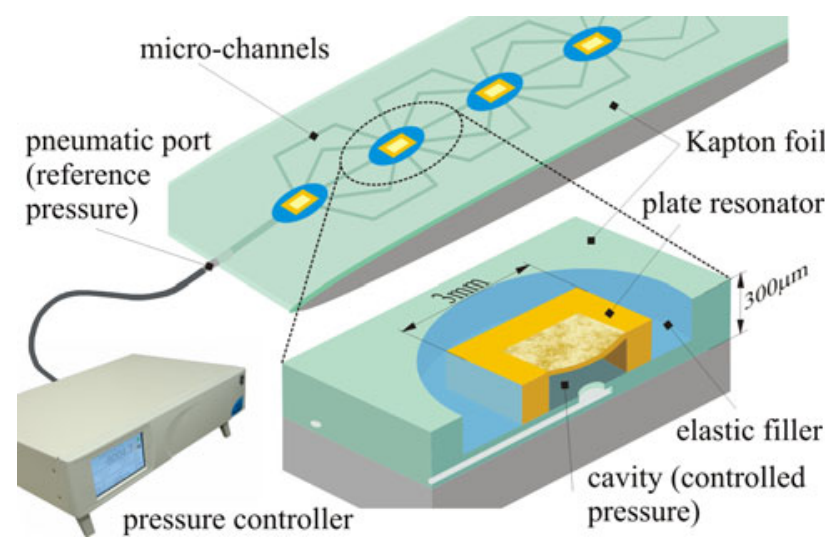

Fig. 2 Sensor design and packaging on a thin structured Kapton foil

(cf. Fig. 1). The sensing elements are thin clamped silicon diaphragms, whose resonance frequencies change with pressure load. The individual sensors are embedded in a thin Kapton carrier foil to facilitate interfacing and stressfree attachment (cf. Fig. 2). No power supply or electronic signal conditioning is required on the sensor side. Maximum sensitivity is achieved by using a differential pressure sensing technique. A single pneumatic supply line is integrated into the Kapton carrier and applies an internal reference back-pressure to all sensing elements in parallel. A pressure controller adjusts the reference pressure to compensate temperature-induced variations.

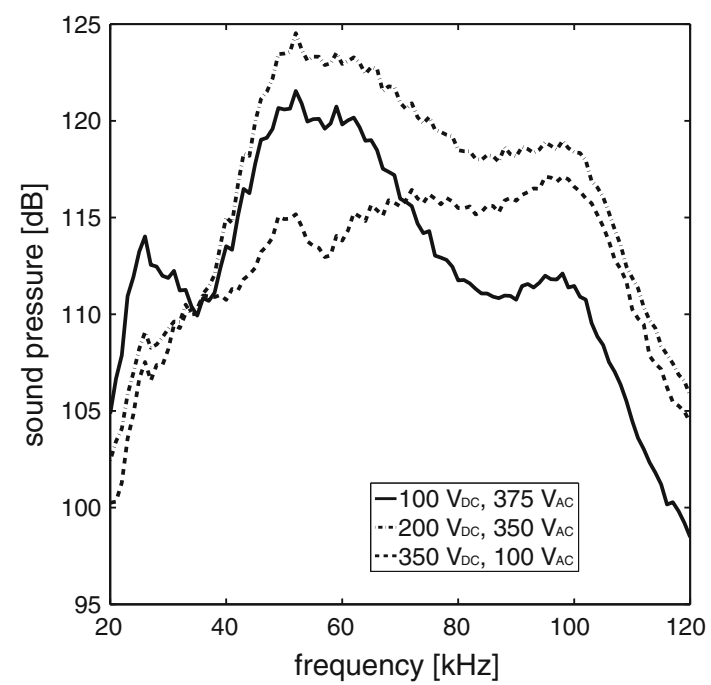

Fig. 3 Transmitting characteristics of the CUT (SensComp 600 Series CUT, $\varnothing 40 \mathrm{~mm}$ ) for different operating voltages (sound pressure level measured $1 \mathrm{~m}$ away from transducer)

The resonance frequency of the clamped silicon diaphragm is used as a measure for the applied pressure difference. This quantity is analyzed rather than the static diaphragm deflection, because of the need for a robust optical readout over comparatively large distances in the presence of significant relative motions and vibrations. The frequency varies as function of the diaphragm's quasistatic deflection caused by the differential pressure load and depends on the diaphragm size, thickness, and material (Fabula et al. 1997; Blevins 2001). For typical sensor dimensions of about $3 \mathrm{~mm}^{2}$ and single-crystal silicon, an operating frequency range between 30 and $150 \mathrm{kHz}$ results (dependent on the considered vibration mode). The corresponding mean pressure sensitivity is approximately $3 \mathrm{~Hz} / \mathrm{Pa}$.

Specific vibration modes of the diaphragm are excited by a capacitive ultrasound transducer (CUT), emitting band-limited "pink" noise. Using ultrasound allows for a wireless, remote excitation of several sensors in parallel without altering the sensing behavior. A CUT is best suited for the present application because of its high transmitting power, high transmitting efficiency in air, and its broad band transmitting characteristics (cf. Fig. 3 and (Manthey et al. 1992; Rafiq et al. 1991)). Experiments have shown that sufficiently strong excitation up to $150 \mathrm{kHz}$ can be achieved using the present CUT (cf. Sect. 4). Interaction between the ultrasound and the flow boundary layer (BL) is assumed to be negligible at frequencies above $30 \mathrm{kHz}$ (Zaman et al. 1987). The same holds true if the interaction between the resonator acting as a zero-mass flux actuator and the BL is considered (Seifert et al. 2004). The impact of environmental vibrations or noise on the sensing 
behavior is equally minimized for resonator operation in the ultrasonic frequency range.

A modulated optical signal with a specific frequency arises from the interference between the sensor surface reflections and a reference illumination, which is derived from the same coherent light source. The detection of signal frequencies beyond $30 \mathrm{kHz}$ is not possible using standard camera technology. It becomes feasible with a smart pixel detector array (Heliotis, Switzerland), which performs a dual-phase lock-in detection on each pixel with a maximum demodulation frequency of $250 \mathrm{kHz}$ (Beer et al. 2005). This detector can acquire images with a resolution of $144 \times 90$ pixels at a frame rate of $6 \mathrm{kHz}$. A high sensitivity of $86 \mathrm{~dB}$ is obtained using an electronic background light suppression at the pixel level.

The resonator is prestressed by selecting a predefined reference back-pressure of less than 100 mbar below the ambient value. The initial loading of the diaphragm enables signed pressure measurements and maximizes the pressure sensitivity. A further benefit is the possibility to perform an in-situ calibration of the sensor array during wind-off conditions. During calibration, the sensors' load is varied by modifying the reference back-pressure within the load range of interest, while the outside pressure remains constant at atmospheric level. A sensor loaded in this way, however, exhibits a different vibration behavior from the one loaded on the outer side (measurement configuration). The resulting systematic measurement error can either be neglected or corrected if necessary.

The optical interrogation of multiple sensors in parallel requires that the target surfaces scatter the incident light evenly into a broad angle. The highly specular top diaphragm surfaces (silicon wafer) are roughened in order to obtain the required diffuse scattering property (cf. Sect. 3).

\section{Sensor design and fabrication}

Single-crystal silicon (SCS) is chosen as material for the resonant device, because of the excellent material properties of SCS and the mature micromachining fabrication technologies. SCS has a strength comparable to that of steel and an elastic behavior up to fracture, leading to minimal hysteresis (Sugiyama et al. 1997).

The fabrication is based on deep reactive ion etching from the rear side of a silicon-on-insulator (SOI) wafer. The insulator of such a wafer acts as a precise etch stop (cf. Fig. 4). Hence, a uniform and reproducible diaphragm thickness can be obtained. Due to the highly anisotropic etching characteristic of common dry etching processes, the sensor cavity features almost vertical walls. The current prototype is designed with a height of $400 \mu \mathrm{m}$ instead of the optimal value of $200 \mu \mathrm{m}$, in order to simplify the wafer

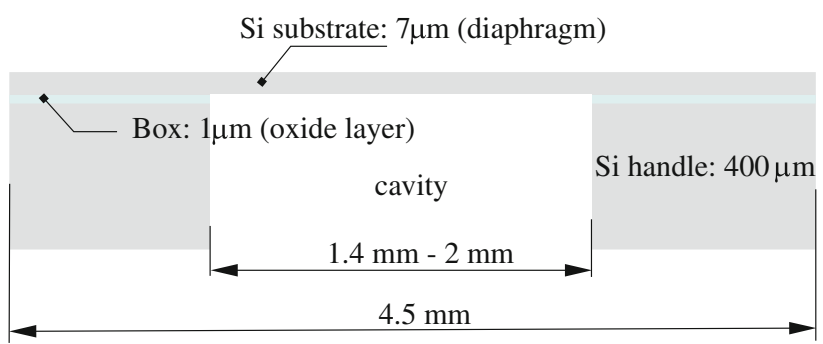

Fig. 4 Sensor layout of the prototype based on a SOI wafer

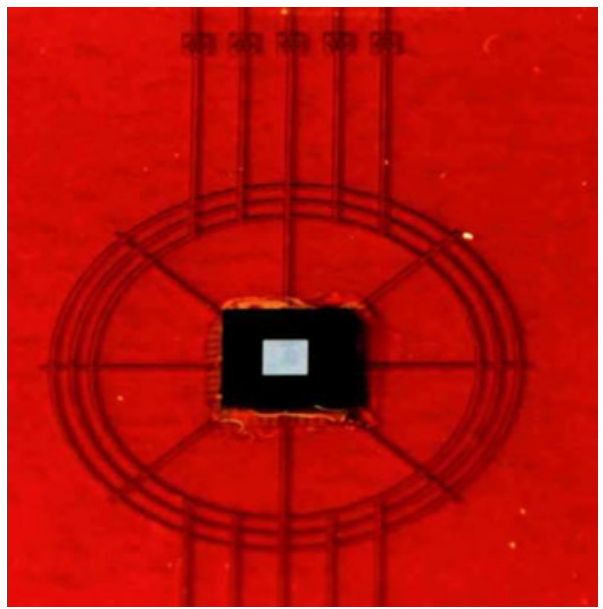

Fig. 5 Plate resonator mounted on a laser structured Kapton carrier. The rough diaphragm surface appears colorless under white light illumination

handling. Four different sensor dies are manufactured with a square diaphragm of $7 \mu \mathrm{m}$ thickness and different edge lengths between 1.4 and $2 \mathrm{~mm}$. The overall chip size of $4.5 \mathrm{~mm}$ assures a strong support of the diaphragm. The sensor is mounted onto the laser structured Kapton carrier using an elastic silicon filler material (cf. Fig. 5). Note the roughened top surface of the membrane, which appears colorless under white light illumination, indicating highly diffuse scattering and low optical dispersion and diffraction.

This optical appearance can be linked to statistical properties of a rough surface such as the rms roughness height $\sigma$ and the spatial autocorrelation length $l_{c}$ (Bennet et al. 1989). The angular range of diffuse scattering increases with increasing $\sigma$ and decreasing $l_{c}$. In order to suppress diffraction phenomena, $\sigma$ should not exceed the wavelength of the interrogation light $(\lambda<800 \mathrm{~nm})$ (Lalanne et al. 1999). The maximum allowable roughness height is furthermore limited by the decrease in the mechanical strength of the silicon diaphragm. Henning et al. (Henning et al. 2004) state a degradation of the fracture strength of SCS for $\sigma$ larger than $100 \mathrm{~nm}$, reaching almost $70 \%$ at $1 \mu \mathrm{m}$ roughness height. Hence, the target surface topography should consist in densely 


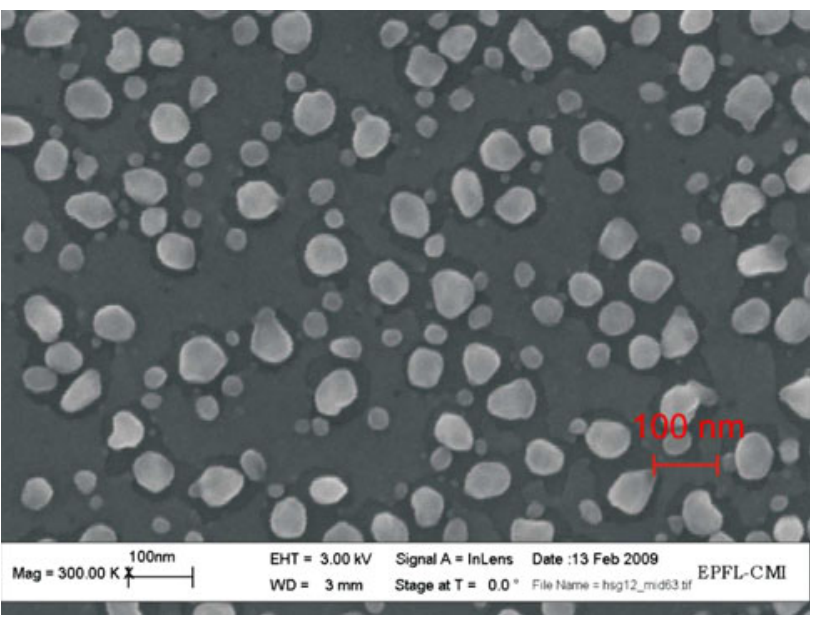

Fig. 6 HSG distribution after poly-silicon deposition and in-situ annealing. Image taken with a scanning electron microscope (SEM)

packed roughness elements with dimensions smaller than $200 \mathrm{~nm}$.

Thermal stresses due to the bi-metal effect can be caused by the deposition of different materials onto the wafer surface (Stemme 1991). They can be avoided by imprinting the roughness pattern directly into the silicon. A novel fabrication process is introduced in the present work to create the required surface properties (Prochazka 2011). It is based on thin film deposition of poly-silicon, which can show a highly non-uniform deposition characteristic for certain process parameters (Ecoffey et al. 2002). The formation of hemispherical poly-silicon grains (HSG) on the mask layer $\left(\mathrm{SiO}_{2}\right.$ film $)$ defines the mask windows with the desired size and random distribution (cf. Fig. 6). After the mask is structured, the roughness is obtained by anisotropic wet etching of SCS and a subsequent stripping of the oxide film. The resulting surface topography consists of a dense and random arrangement of grooves with a pyramid geometry (walls angled $54.74^{\circ}$ with respect to the wafer surface) and a lateral extent of less than $200 \mathrm{~nm}$ (cf. Fig. 7).

\section{Resonator vibration behavior}

Tests were carried out in a temperature and pressure controlled test cell (cf. Fig. 8) to verify the sensing behavior of the MEMS resonators. The gas temperature was set and controlled using a Peltier heater and two heat exchangers between 20 and $30^{\circ} \mathrm{C}$ (control accuracy $< \pm 0.02^{\circ} \mathrm{C}$ ).

The sensor was loaded by varying the pressure in the back cavity (reference back-pressure) using a fast and highly accurate pressure controller (PACE 5000, GE Sensing, control accuracy $< \pm 10 \mathrm{~Pa}$ ). The pressure on the sensor front surface, being exposed to the test cell, was not

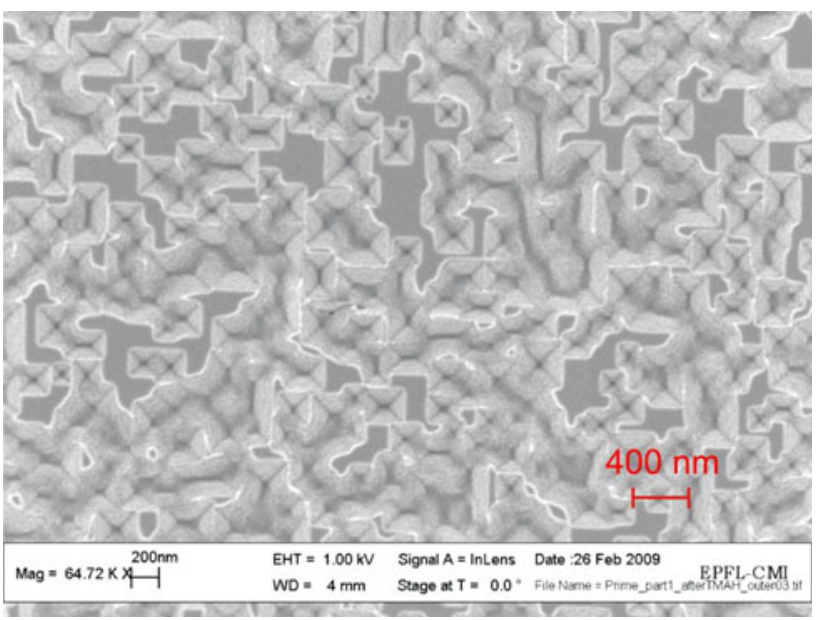

Fig. 7 SEM image of the roughness topography after oxide stripping

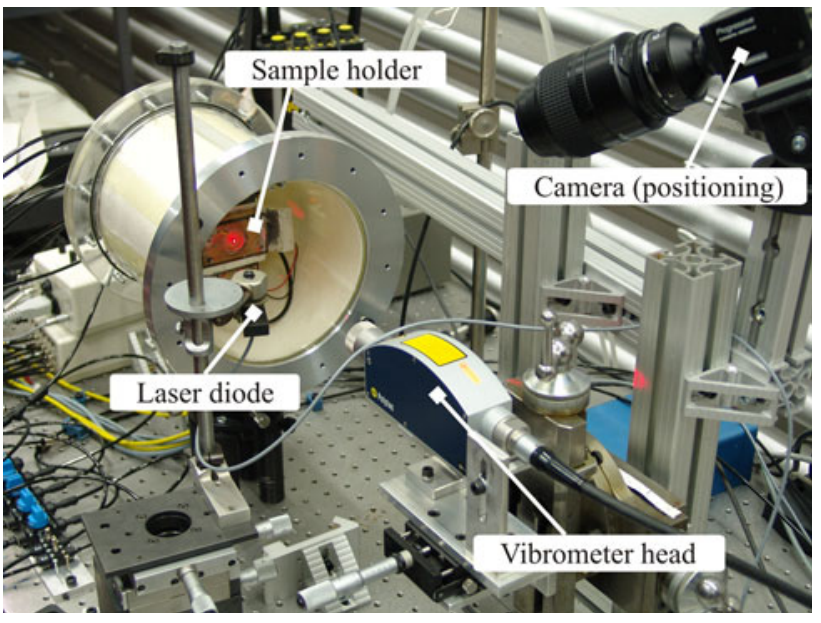

Fig. 8 Experimental setup for the investigation of the sensor dies using optical excitation

adjusted, but kept at ambient conditions. As long as the pressure value on the corresponding side of the diaphragm does not deviate by more than $\pm 5 \mathrm{kPa}$ from the real conditions, pressure dependent mass-loading effects have a negligible influence on the vibrating behavior of the resonator. For higher pressure deviations, the shift in resonance frequency exceeds the specified measurement uncertainty, and the data based on the simplified loading procedure have to be corrected (Prochazka 2011).

\subsection{Optical and acoustic excitation}

Due to the limited space inside the test cell, acoustic excitation cannot be applied for investigations where temperature adjustment and control are required. Instead, an external pulsed laser diode (Flexpoint $10 \mathrm{~mW}$, Laser Components $\mathrm{GmbH}$ ) was used as an excitation source. The absorbed laser light locally heats the diaphragm and 
Fig. 9 Six structural vibrating mode shapes on a square clamped diaphragm (based on FEM computations using ANSYS)

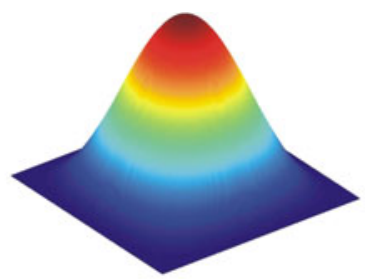

$(1,1)$

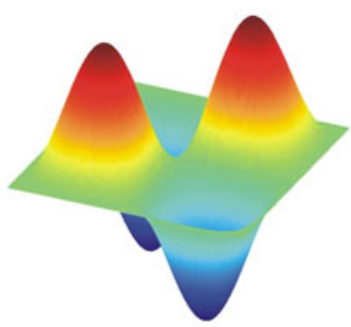

$(2,2)$

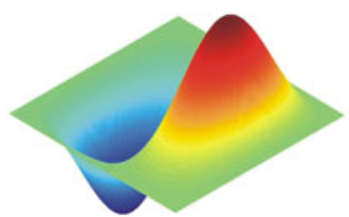

$(1,2)$

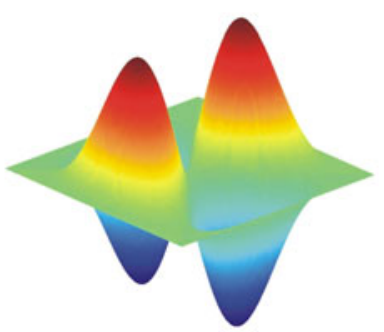

$(1,3)$

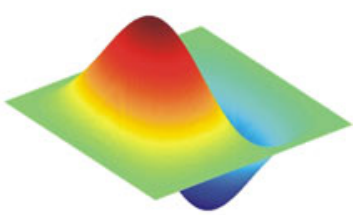

$(2,1)$

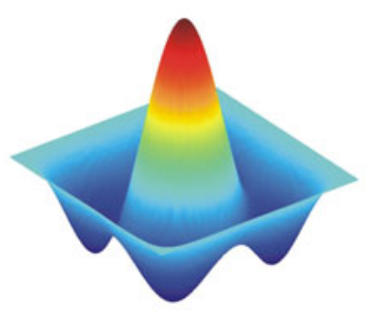

$(3,1)$ induces thermal stresses, which drive the resonant structure with the pulse repetition frequency (Lammerink et al. 1991; Uttamchandani et al. 1987). The vibration amplitude was recorded using a single-point laser Doppler vibrometer (OFV-551, Polytec GmbH) situated outside of the test cell. The positions of the interrogation and excitation laser beams on the diaphragm surface were adjusted so as to maximize the vibrometer output signal.

The spectral response of the diaphragm was computed using a Fourier analysis algorithm. During a test sequence, the excitation frequency was swept across the range of interest with minimum increment of $20 \mathrm{~Hz}$ in the neighborhood of the resonance peak in the amplitude spectrum. All structural vibration modes in the frequency range from 30 to $150 \mathrm{kHz}$ (restricted by the operating range of the CUT) were considered. The corresponding mode shapes as computed with the finite element modeling (FEM) software ANSYS are shown in Fig. 9.

Compared to acoustic noise excitation, the single-frequency optical excitation provides a better analysis of the oscillating behavior regarding the damping behavior (Q-factor) and interference phenomena caused by fluid structure coupling. The quality factor can easily be determined from the amplitude spectrum based on the definition $Q=f_{\text {res }} / \Delta f_{3 d B}$ where $f_{\text {res }}$ and $\Delta f_{3 d B}$ denote the resonance frequency and the band width of the amplitude spectrum, respectively (Stemme 1991).

Mode-selective optical excitation of the resonant structure is performed by appropriate positioning of the driving force (i.e., the focused laser beam), taking care to prevent coupling between different modes. Acoustic excitation, on the other hand, does not rely on point excitation, but still can drive certain higher modes by narrow-band acoustic noise. For measurements in this configuration, the test cell was kept open, with the sound source (CUT) located 1 meter away. The diaphragm oscillation induced by acoustic noise was recorded and analyzed again using the vibrometer.

\subsection{Mode-specific sensing behavior}

The operating lines (i.e., resonance frequency as function of the pressure load) for all considered modes are illustrated in Fig. 10, as determined using both optical (solid lines) and acoustic excitation (markers). Dependent on the mode considered, the frequency band of the acoustic noise was adapted to the corresponding operating frequency range of the resonator (cf. shaded area for mode $(2,2)$ in Fig. 10). Even though the noise spectrum is kept narrow, coupling between neighboring modes occurs for certain sensor loads smaller than $14 \mathrm{kPa}$. While only the fundamental $(1,1)$ and the $(3,1)$ mode can be driven by acoustic noise excitation across the whole pressure range, all modes are addressable using optical excitation. Considering the intermediate modes, acoustic excitation is only applicable if no overlap between neighboring operating lines occurs. Within the overlap region, the mode more receptive for acoustic sound dominates the vibrating condition. The $(3,1)$ mode dominates over the $(1,3)$ and the $(2,2)$ mode. The $(1,2)$ mode is in fact more receptive than the $(2,2)$ mode, but less receptive to sound compared to the fundamental mode.

In order to explain the mode-dependent receptivity of a plate resonator, its damping behavior as expressed by the quality (Q-) factor can be analyzed (cf. Fig. 11). In addition to the overall losses of the plate resonator obtained from tests with optical excitation, the damping effect caused by acoustic radiation alone is considered as well. This loss mechanism relies on sound waves generated by the oscillating plate and radiated into the far field (Crocker 1998). 


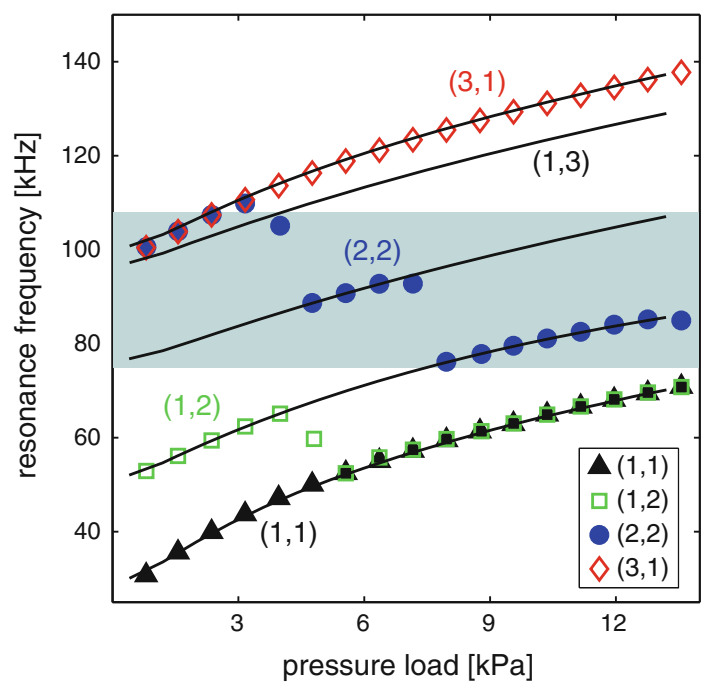

Fig. 10 Operating lines corresponding to the first 6 vibrating modes obtained with optical (solid lines) and sound excitation (markers). Diaphragm size: $1.8 \mathrm{~mm}$. Shaded area Frequency band of acoustic noise used for the excitation of mode $(2,2)$

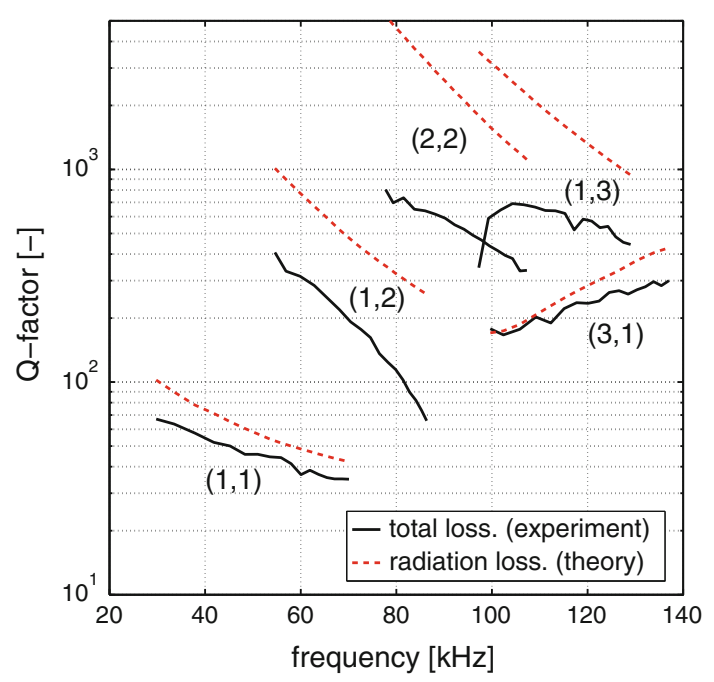

Fig. 11 Damping behavior (Q-factor) as function of the frequency for all considered modes. Solid lines represent the experiment and the dashed lines the theory. Diaphragm size: $1.8 \mathrm{~mm}$

Acoustic sound radiation depends on the fluid properties, the size of the vibrating structure, and the surface velocity distribution. The dependence on the modal shape is clearly illustrated in Fig. 11 (dashed lines). The data are obtained by solving numerically (Matlab) the Rayleigh integral, which describes the sound pressure field induced by a vibrating structure of an arbitrary geometry (Lemmen et al. 1996).

Considering first the overall losses, one may conclude that the fundamental mode is highly damped $(\mathrm{Q}<50)$ followed by the $(1,2)$ and $(3,1)$ modes with Q-factors above 100 . The $(2,2)$ and $(1,3)$ modes exhibit the lowest damping with Q-factors well above 200. For all modes except the highest mode $(3,1)$, where the opposite effect is apparent, the damping increases with increasing frequency. The overall damping of the plate resonators qualitatively agrees with the damping characteristics caused by acoustic radiation. A symmetrical mode shape seems to provoke strong radiation of sound waves, whereas modes with an asymmetrical shape are poorer sound emitters (Stemme 1991; Tudor et al. 1997). Mode (3,1), which can also be characterized as a symmetrical mode, confirms this conjecture. The residual loss sources, which could explain the discrepancy between theory and experiment, are not conclusively identified. Clamping losses could form a considerable contribution to the overall losses for unbalanced modes such as the fundamental one (Stemme 1991). By comparison, the damping caused by the closed pressurized reference cavity should be smaller for a symmetrical than for an asymmetrical mode (Beltman et al. 1998). No significant influence of the microchannels on the damping can be seen in the experiments.

Based on the principle of reciprocity, one can infer that a good radiator is also a good receiver for sound (Fahy et al. 2007). The fundamental and the (3,1) modes show higher damping by acoustic radiation than all neighboring modes situated in the same excitation frequency band. Hence, both modes are also efficient receivers for sound and dominate the vibration of the resonator over the whole pressure range. The asymmetrical modes $(2,2)$ and $(1,3)$ are the worst transmitters and receivers for sound and cannot be considered for the present application.

The influence of damping on the measurement performance is quantified by considering the standard deviation of 20 subsequent measurements for all modes of vibration (cf. Fig. 12). The tests are based on acoustic excitation and an ordinary Fast Fourier Transform as demodulation algorithm. If one assumes the required system sensitivity of $20 \mathrm{~Pa}$ (cf. Table 1) and an average pressure sensitivity of the resonator of $3 \mathrm{~Hz} / \mathrm{Pa}$, a maximum allowable uncertainty of $60 \mathrm{~Hz}$ must be achieved (i.e., $30 \mathrm{~Hz}$ standard deviation). The values for the highly damped fundamental mode lie far above this limit, while the $(3,1)$ mode shows a considerably better measurement performance. Only mode $(1,2)$ fulfills the required measurement uncertainty, but it can only be utilized for sensing within a limited pressure range $(<4 \mathrm{kPa}$, cf. Fig. 10). Thus mode $(3,1)$ best meets the requirements of the present application regarding the measurement range and performance.

\subsection{Measurement sensitivity}

A plate resonator designed for the specified measurement range and performance exhibits a pronounced non-linear sensing characteristic (cf. Fig. 13). The unloaded sensor 


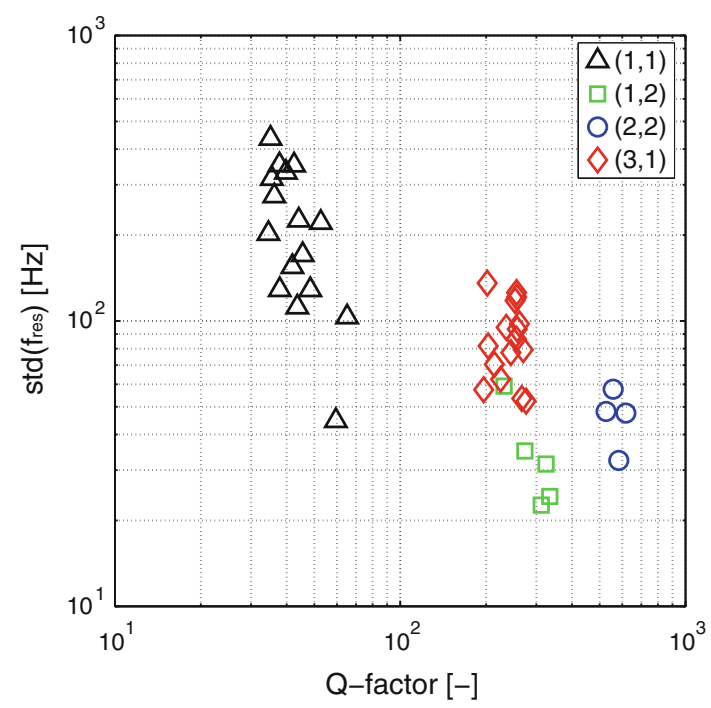

Fig. 12 Measurement uncertainty versus Q-factor for all modes, which can be driven by acoustic excitation. The uncertainty is defined as the standard deviation of 20 subsequent measurements

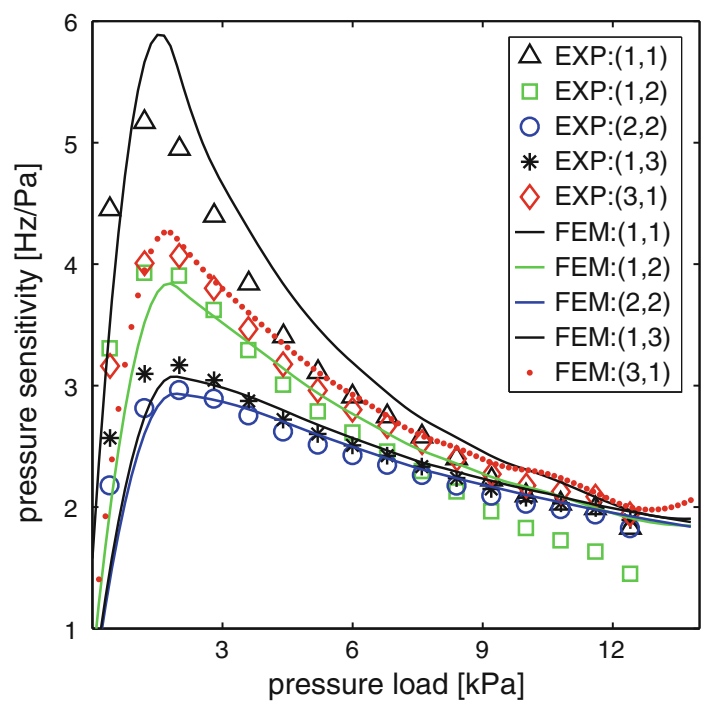

Fig. 13 Pressure sensitivity versus pressure load for all considered modes (optical excitation) and comparison with FEM simulations using ANSYS (diaphragm vibrating in vacuum), Diaphragm size: $1.8 \mathrm{~mm}$

shows a very poor pressure sensitivity, which increases strongly if slightly loaded. Beyond the maximum value, the sensitivity decreases continuously with increasing load. A good agreement between experiment and numerical simulations can be observed. The FEM simulations performed in ANSYS are based on a clamped plate vibrating in vacuum (Prochazka 2011).

The non-linear sensing behavior requires a multi-point calibration, which can easily be performed in-situ using the present system. If the measurement range of a specific application is approximately known, the sensing

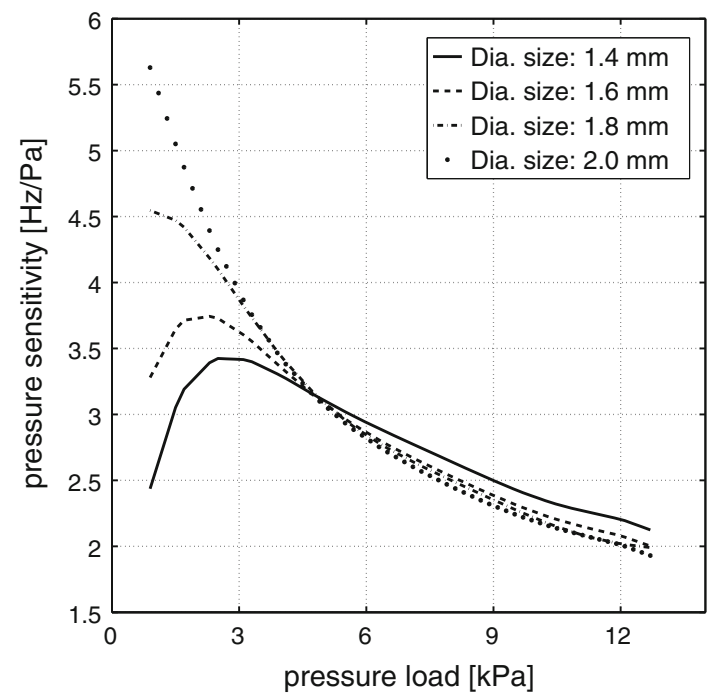

Fig. 14 Influence of the diaphragm size (edge length of a square diaphragm) on the pressure sensitivity for mode $(3,1)$

performance can be maximized by selecting an appropriate reference back-pressure (i.e., initial operating point) in order to operate the resonator within a high sensitivity range. Comparing the pressure sensitivity of the individual modes, significant differences exist only in the low pressure load range $(<6 \mathrm{kPa})$. The fundamental mode shows the highest sensitivity with a maximum value higher $5 \mathrm{~Hz} / \mathrm{Pa}$ followed by modes $(1,2)$ and $(3,1)$ with approximately $4 \mathrm{~Hz} / \mathrm{Pa}$. Modes $(2,2)$ and $(1,2)$ exhibit the lowest maximum sensitivity of approximately $3 \mathrm{~Hz} / \mathrm{Pa}$. At higher pressure loads, the sensitivity is about the same for all modes.

The size of the diaphragm shows also a significant influence on the pressure sensitivity, but again only for sensor loads $<4 \mathrm{kPa}$ (cf. Fig. 14). Within that range, the sensitive of a resonator operating in mode $(3,1)$ decreases and the point of maximum sensitivity moves to higher pressure loads with decreasing sensor size.

The cross-sensitivity of the resonator to temperature variations was verified on a Kapton carrier containing five sensing elements. Operating lines of all sensing elements were determined at 20,24 , and $28^{\circ} \mathrm{C}$. Based on these data, the temperature sensitivity was evaluated as an average value for a temperature range between 20 and $24^{\circ} \mathrm{C}$ (cf. solid markers in Fig. 15) and the range between 24 and $28^{\circ} \mathrm{C}$ (hollow markers). Only results for mode $(3,1)$ are shown. Mode $(1,2)$, which also suits for pressure sensing within a limited pressure range, exhibits a similar behavior as mode $(3,1)$. Except for a slightly loaded sensor, the cross-sensitivity is well below $30 \mathrm{~Hz} / \mathrm{K}$ across the whole considered pressure range. If expressed with respect to a measurement range of $10 \mathrm{kPa}$ and assuming a mean pressure sensitivity of $3 \mathrm{~Hz} / \mathrm{Pa}$, a value smaller than $0.1 \% / \mathrm{K}$ results. The different sign of the sensitivity between the 


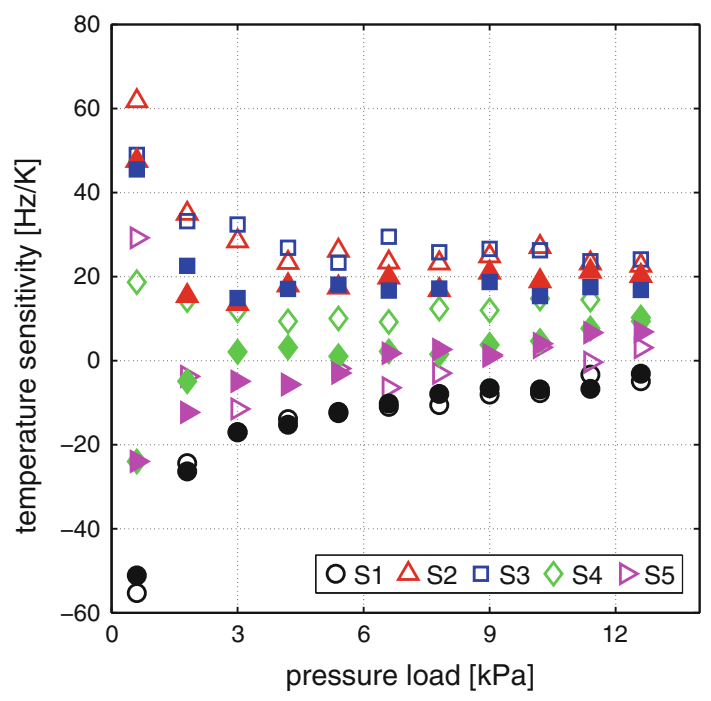

Fig. 15 Temperature cross-sensitivity for mode $(3,1)$ averaged over a temperature range between 20 and $24^{\circ} \mathrm{C}$ (empty markers) and between 24 and $28^{\circ} \mathrm{C}$ (full markers)

individual sensing elements points to a remaining crossinfluence of the mechanical mounting inside the test cell.

\section{Wind tunnel tests}

Wind tunnel tests were carried out in order to verify the resonator behavior in a flow and in a highly vibrating environment. Reference measurements with a conventional pressure tap system were compared to vibrometer read-outs of several MEMS sensors.

\subsection{Experiment infrastructure}

The wind tunnel is an open circuit facility of the blower type and provides a flow of high quality (cf. Fig. 16a, (Pompeo 1992)). The test section, equipped with a removable ceiling panel, is $0.4 \mathrm{~m}$ wide, $0.5 \mathrm{~m}$ high, and 0.7 $\mathrm{m}$ long. An open ceiling allows for an optical and acoustic access to the wind tunnel model (cf. Fig. 16b). For sensor interrogation, again the established single-point laser Doppler vibrometer in combination with acoustic excitation was used.

The tests were performed on a NACA 0012 airfoil with a chord of $0.3 \mathrm{~m}$. The airfoil was horizontally mounted between the two side walls of the test section (cf. Fig. 16b). Using an electronic inclinometer, the angle of attack $\alpha$ could manually be adjusted with an accuracy of approximately $0.05^{\circ}$. Five plate resonators (diaphragm size $2 \mathrm{~mm}$ ) were installed on the suction side of the airfoil and distributed along the chord. They were arranged on a single carrier foil with a length and width of 240 and $40 \mathrm{~mm}$, respectively (cf. Fig. 16b). The carrier contains the pneumatic tubing system with $50 \mu \mathrm{m}$ deep and $200 \mu \mathrm{m}$ wide, laser structured microchannels connecting the individual sensor cavities (cf. Fig. 16c, layer 1). Due to the small model dimensions and the comparatively large sensor height of the prototype, the 0.6 -mm-thick sensor stripe was
Fig. 16 Measurement setup: a Sketch of the boundary layer wind tunnel at IFD (ETHZ). b Test section with the instrumented NACA 0012 airfoil and the LDV head (view from the top through the open ceiling). c CAD sketch (detail) of the sensor stripe design and the pneumatic tubing system layout

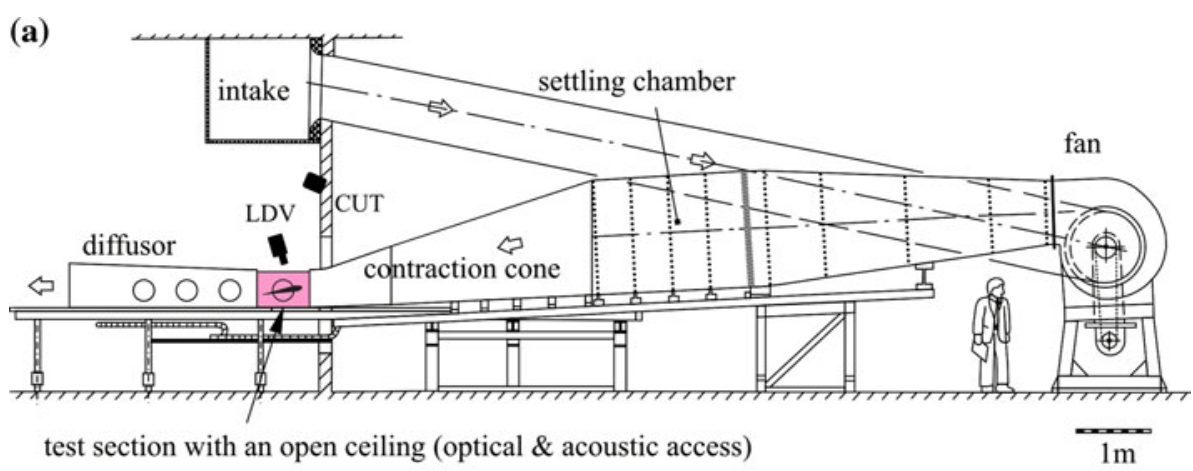

(b)

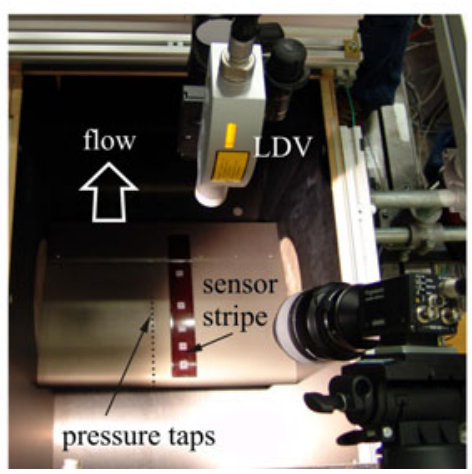

(c)

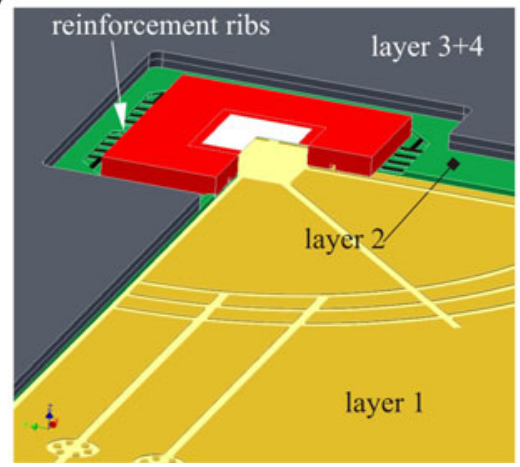


recessed in a milled slot to prevent any flow disturbances by the sensor unit. For reference measurements, 29 pressure taps were installed adjacent to the resonant sensors (lateral displacement $30 \mathrm{~mm}$ ). The read-out was performed using a mechanical pressure scanner providing 48 ports (Scanivalve Corp., model 48J9) and a pressure transducer of type PDCR 22 (GE Druck Limited) with a measurement range of $7 \mathrm{kPa}$ and a measurement uncertainty of $10 \mathrm{~Pa}$. The reference pressure values at the resonators' location were determined by linear interpolation between the data of neighboring taps. The vibrometer head was positioned outside the flow, approximately $0.5 \mathrm{~m}$ above the model. For each sensor and angle of attack, the detector and the ultrasound transducer were adjusted, so as to maximize the interrogation signal.

For demodulation of the vibrometer output signal, the Welch method was applied (Hayes 1996), which averages multiple Fourier spectra. A rectangular window, a segment length of 500 and an overlap of 50\% was used for the algorithm as implemented in Matlab. In total, 100 ksamples acquired with $500 \mathrm{kHz}$ sample rate were used for processing. Averaging over 20 subsequent measurements was performed. All tests were carried out on two sensing elements S1 and S2 located at $x / c=0.1$ and 0.2 , where $x$ denotes the distance between the sensor and the leading edge of the airfoil and $c$ the wing's chord. Free-stream velocities $U_{\infty}$ between 12.5 and $35 \mathrm{~m} / \mathrm{s}$ and an angle of attack $\alpha$ between 0 and $16^{\circ}$ limit the operating range of the airfoil. The corresponding chord Reynolds numbers $R e_{c}=U_{\infty} c / v$ range from $2.5 \cdot 10^{5}$ to $7 \cdot 10^{5}$. The reference pressure in the cavity of the resonator was kept constant at $-3 \mathrm{kPa}$ during measurement. Only mode $(3,1)$ was utilized in the present tests.

The impact of environmental vibrations on the sensing behavior was investigated by considering the standard deviation (i.e., measurement uncertainty) of the pressure data obtained from 20 subsequent measurements (cf. Fig. 17). Although the environmental vibrations are considerably larger at higher flow speeds (or $R e_{c}$ ), the measurement uncertainty remains smaller than $20 \mathrm{~Pa}$ over the whole considered operating range of the airfoil. The rising uncertainty at $16^{\circ}$ angle of attack and higher $R e_{c}$ is caused by high pressure fluctuations within the fully separated flow on the wing.

\subsection{Measurement comparison and error analysis}

A comparison between the measurement system under investigation and the reference technique revealed a systematic measurement error, which exceeds the maximum allowable measurement uncertainty of $20 \mathrm{~Pa}$ for certain operating conditions of the airfoil (cf. Fig. 18). The error in Fig. 18 is expressed as the absolute discrepancy between

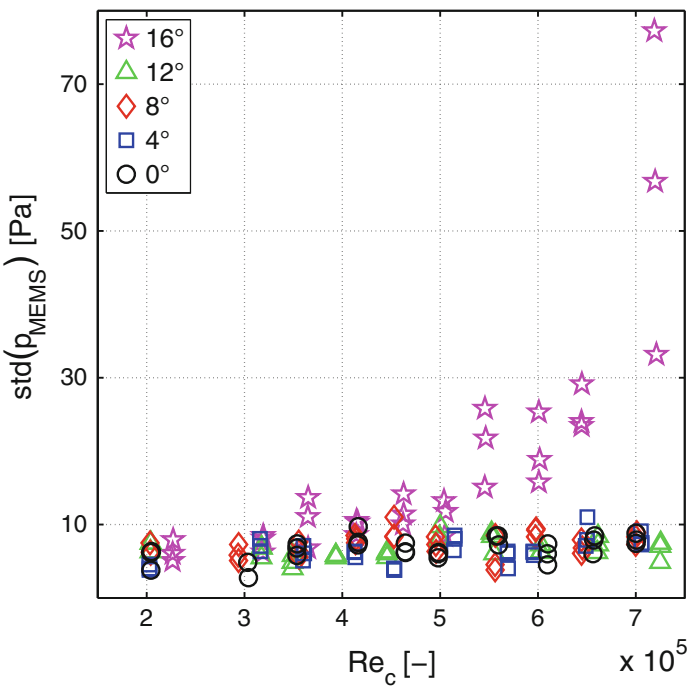

Fig. 17 Influence of environmental vibrations on the sensing behavior: Standard deviation of 20 subsequent measurements on S1 versus chord Reynolds number. Signal demodulation is based on the Welch algorithm

the pressure data acquired using the two measurement systems. It is represented as a function of the tap pressure $p_{\text {tap }}$ and the pressure gradient in streamwise direction $d p_{\text {tap }} / d x$ at resonators location. An increasing negative wall pressure for a constant $\alpha$ goes along with an increasing flow speed or $R e_{c}$ from $2.5 \cdot 10^{5}$ to $7 \cdot 10^{5}$. The left figures represent the data for $\mathrm{S} 1$ and on the right the results for $\mathrm{S} 2$ are shown.

The error increases with increasing $R e_{c}$ and pressure gradient, but also shows a pronounced dependency on the angle of attack. For fully separated flow conditions at $16^{\circ}$ angle of attack, the error is significantly reduced. The strong variation of the error at high $R e_{c}$ and fully separated flow is caused by the existing intense pressure fluctuations (cf. Fig. 17). Further interesting findings are the less pronounced measurement error obtained at S2 compared to S1 and the positive sign of the error for all $\alpha$ at $S 2$. In contrast, S1 provides a negative error for 0 and $4^{\circ}$ angle of attack and a positive one for higher $\alpha$. Measurement errors, which may arise on the pressure tap side such as developing eddies in the cavity formed by the tap or flow turbulence, should be smaller than $1 \%$ of the dynamic pressure, i.e., $\ll 10 \mathrm{~Pa}$ (Shaw 1960; Bradshaw et al. 1968). Based on these findings, the measurement discrepancy seems to stem from an interference between the flow and the resonator. A pressure gradient on the considered wing of up to $20 \mathrm{~Pa} / \mathrm{mm}$ could modify the vibrating behavior due to a non-uniform load distribution on the 2-mm large resonant structure. The still existing dependency on $\alpha$ in Fig. 18 indicates an influence of another flow parameter. The wall shear stress $\tau_{\text {wall }}$ induced in the boundary layer depends on different parameters such as the flow state (laminar or 

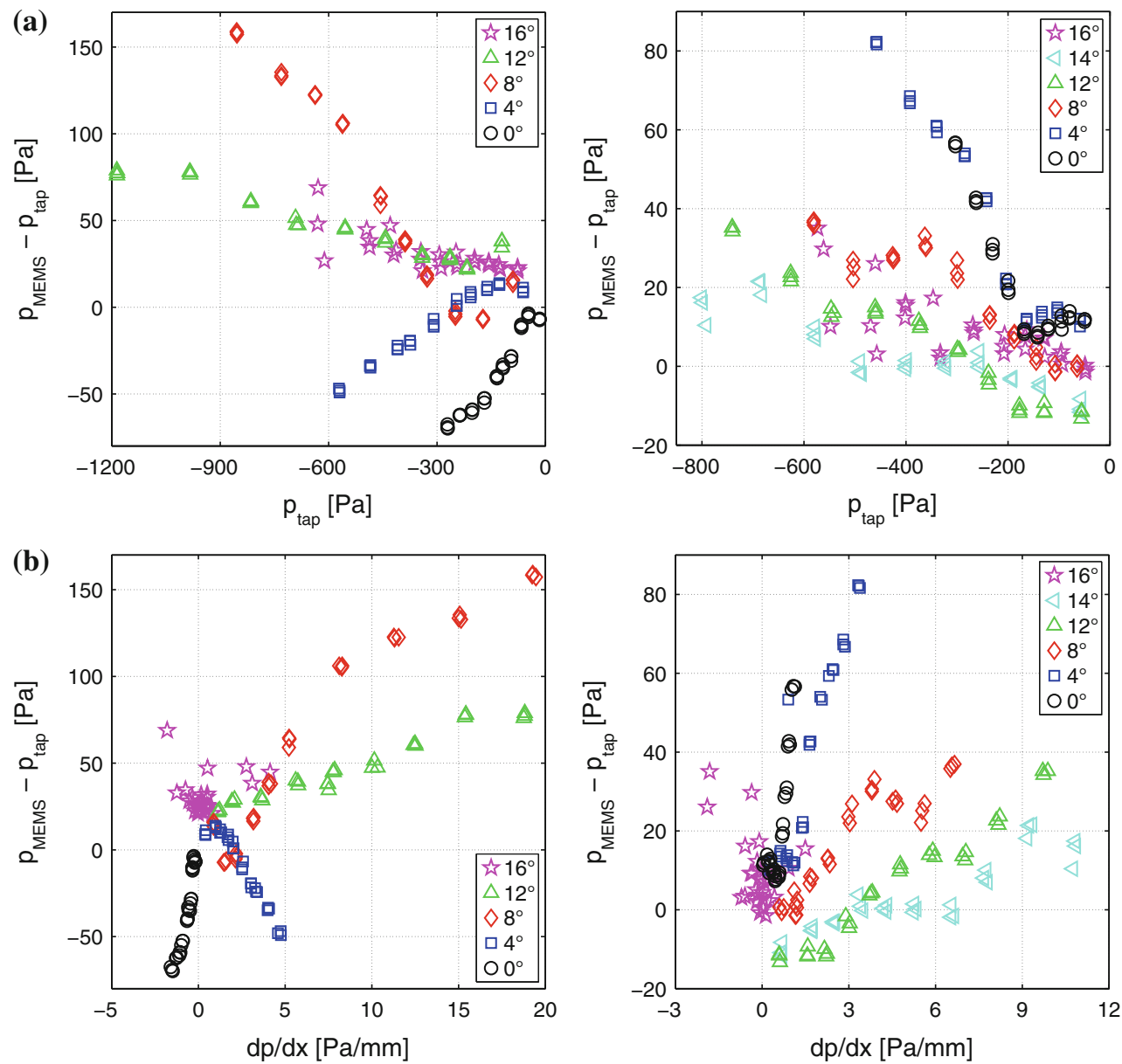

Fig. 18 Absolute measurement discrepancy between MEMS and pressure tap for S1 (left) and S2 (right) and different operating conditions of the airfoil ( $\alpha$ and $U_{\infty}$ variation). a Data plotted versus

the tap pressure. b Data plotted versus the pressure gradient in streamwise direction at sensors location

turbulent), speed, pressure gradient, and the distance from the leading edge (Schlichting et al. 2000). Unless $\tau_{\text {wall }}$ is precisely known a quantitative verification of its impact is not feasible. However, an increasing measurement error with increasing $U_{\infty}$ and a smaller error for S2, which is further downstream compared to $\mathrm{S} 1$, point to an influence of $\tau_{\text {wall }}$ on the vibration behavior. The small measurement error for separated flow conditions at $16^{\circ}$ angle of attack, where $d p / d x$ and $\tau_{\text {wall }}$ vanish, confirms this conjecture.

The different sign of the error obtained with S1 (cf. Fig. 18) should not be related to the sign of the pressure gradient. At zero angle of attack, a favorable and at $4^{\circ}$, an adverse pressure gradient is present at S1 location (cf. Fig. 18). In order to verify if the boundary layer (BL) state induces the change in sign, tests with a laminar and a turbulent $\mathrm{BL}$ at the resonator and tap location were performed (cf. Fig. 19). The study was conducted on S1 and $\alpha=0^{\circ}$ in order to maintain a laminar BL over the whole considered $R e_{c}$ range. Transition between a laminar and a turbulent $\mathrm{BL}$ was triggered using a $0.5 \mathrm{~mm}$ high transition strip attached at $x / c=0.03$. In fact, the sign of the error seems to depend on the BL state. Accordingly, the negative sign of the error for $\alpha=0$ and $4^{\circ}$ in Fig. 18 (left) can be associated with a laminar BL at S1 location, whereas for higher $\alpha$, the BL becomes turbulent and the measurement discrepancy positive.

\section{Full-field optical interrogation}

In order to demonstrate the feasibility of parallel interrogation of a large number of sensors using the "activepixel" CMOS camera (SPDA) (cf. Sect. 2), tests were carried out outside the flow environment (cf. Fig. 20). For excitation of the resonator's fundamental vibration mode, again narrow-band acoustic noise was used $\left(f_{\text {band }}=\right.$ $40-100 \mathrm{kHz}$ ). The ultrasound transducer (CUT) was placed $1.5 \mathrm{~m}$ apart from the sensor. From a distance of 


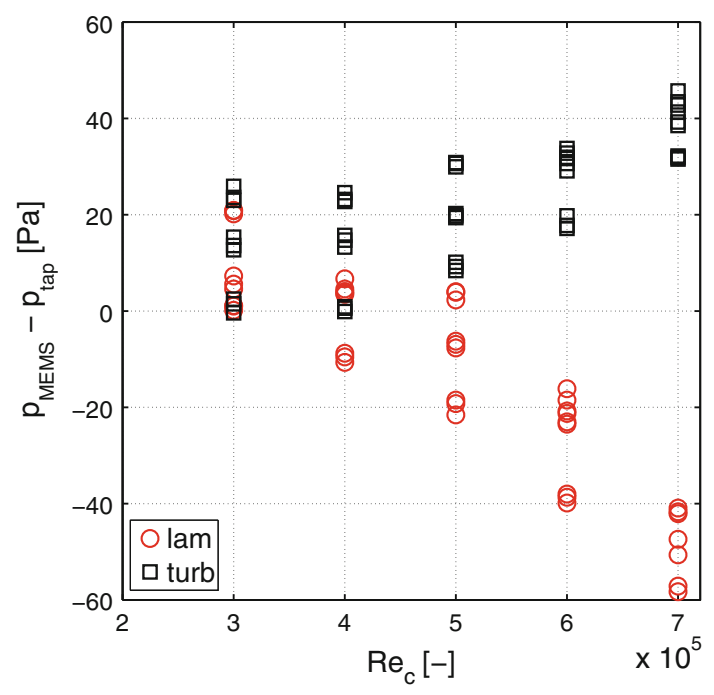

Fig. 19 Absolute measurement discrepancy between MEMS and pressure tap versus $R e_{c}$ for a laminar and a tripped, turbulent BL at S1 location and $\alpha=0^{\circ}$

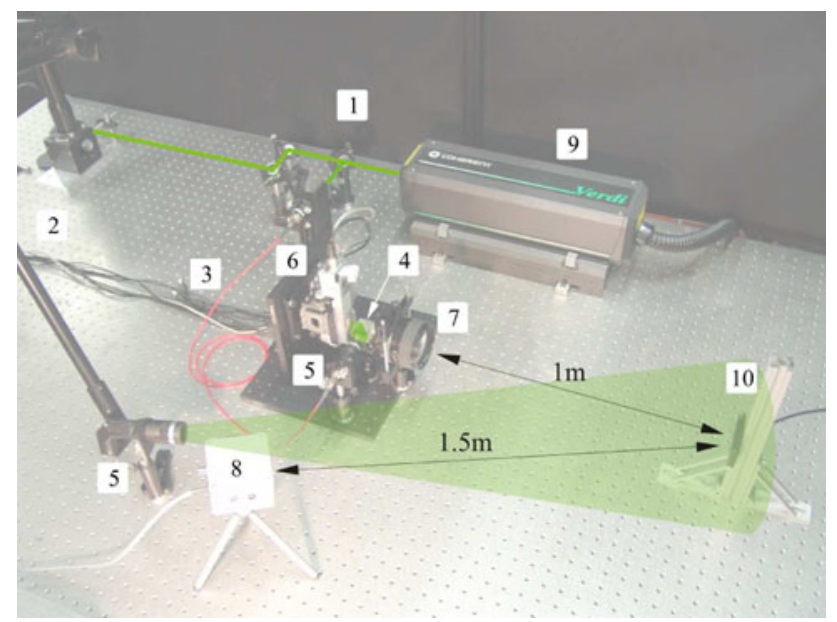

Fig. 20 Setup of the full-field interrogation system: 1 Fresnel beam expander, 2 articulated laser arm, 3 fiber optics delivery system, 4 beam splitter, 5 beam expander, 6 SPDA, 7 lens, 8 ultrasound transducer, 9 laser, 10 sensor

approximately $1 \mathrm{~m}$ and using a standard lens (focal length $85 \mathrm{~mm}), 4$ pixels of the detector array $(144 \times 90$ pixels $)$ were registering the diaphragm signal (cf. Fig. 21). The source beam of a CW laser (Coherent Verdi V5, $\lambda=532$ $\mathrm{nm}$, output power $1 \mathrm{~W}$ ) was split into two parts using a Fresnel beam sampler (cf. Fig. 20). The object beam containing $95 \%$ of the power was expanded to cover a target area of around $0.4 \mathrm{~m} \times 0.4 \mathrm{~m}$. The reference beam was redirected towards the camera using a fiber optic delivery system. A collimator expands the reference beam to cover the detector area. A beam splitter was used to combine the

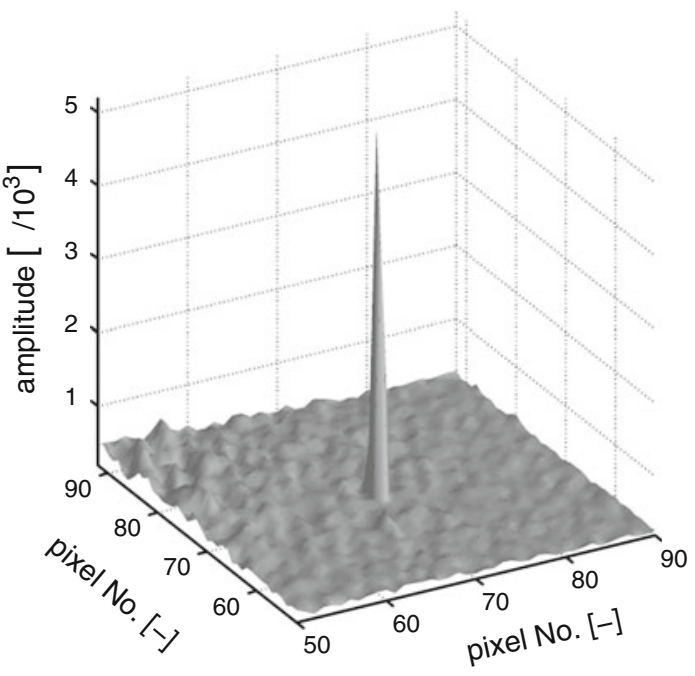

Fig. 21 Demodulated image (lock-in amplitude) obtained from the SPDA at resonance operation and a pressure load of $4.5 \mathrm{kPa}$ (only a cutout of the detector array is shown)

light from the scene and the light from the reference beam. Due to interference, the result of this superposition is a harmonic, intensity-modulated signal superimposed on a constant offset. The demodulation of the ac signal is based on a dual-phase lock-in detection on the pixel level. During the experiment, the resonator was loaded by setting and controlling the back pressure between 0.3 and $11.5 \mathrm{kPa}$ ( $0.5 \mathrm{kPa}$ steps) below the ambient value using the pressure controller.

The demodulation frequency of the SPDA was swept from 50 to $80 \mathrm{kHz}$ in 100 steps (300 Hz per step). A total averaging over 200 measurements was performed per run (constant pressure load). During postprocessing, the raw data were first analyzed to locate the pixel cluster, which corresponds to the diaphragm (cf. Fig. 21). The lock-in amplitude for each frequency step was then extracted from the image data (cf. Fig. 22). The amplitude data within an interval of $8 \mathrm{kHz}$ around the peak value were fitted using the least-squares method with a Gaussian profile. Finally, the resonance frequency was found using a peak detection based on the Gaussian fit.

Considering the operating lines for the fundamental mode, a very good agreement between the data obtained using the single-point vibrometer and the SPDA exists in a pressure load range between 3 and $8 \mathrm{kPa}$ (cf. Fig. 23). Between 8 and $11 \mathrm{kPa}$, a pronounced discrepancy is obvious, which coincides with a strongly damped vibration behavior of the resonator (cf. Q-factor characteristics in Fig. 23). The strong damping is caused by a destructive interference between acoustic modes, which occurred in the outer recess (i.e., open cavity) of the first sample holder version and the fundamental mode of the resonator. After a 


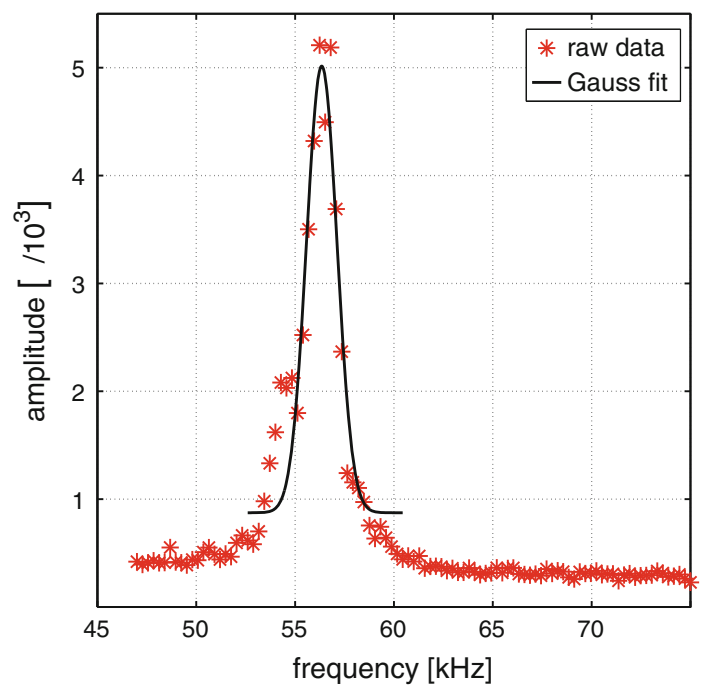

Fig. 22 Signal amplitude after lock-in detection on 1 pixel $(72,20)$, Gaussian fit within an interval of $8 \mathrm{kHz}$ (solid line)

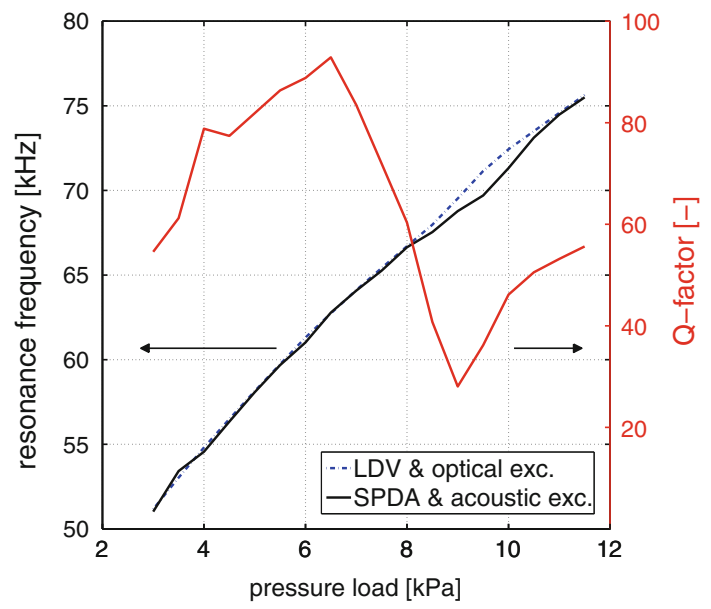

Fig. 23 Operating lines for mode $(1,1)$ acquired using the singlepoint (LDV) and the full-field (SPDA) interrogation method. The Q-factor characteristic (red) is based on LDV measurements and optical excitation

redesign of the outer recess geometry, the fluid-structure interference effect is no longer observed (cf. mode $(1,1)$ in Fig. 11).

\section{Conclusion and outlook}

The MEMS plate resonator shows the expected measurement performance in terms of operating resonance frequency and pressure sensitivity. The fundamental mode $(1,1)$ is highly damped due to acoustic radiation and thus not suitable for pressure sensing, where high sensitivity is required. The $(3,1)$ mode is the most suitable mode of vibration for the present application. The sensor operating in that mode shows much lower damping compared to the fundamental mode and still shows sufficient high receptivity for acoustic noise to be excited over the whole required pressure range. The required measurement accuracy of $20 \mathrm{~Pa}$ is achievable for flow velocities $<20 \mathrm{~m} / \mathrm{s}$. An interaction between the flow and the sensor creates a measurement error, which increases with increasing freestream velocity. It is reasoned that this interaction is caused by the wall shear stress and the pressure gradient induced by the flow. A sensor recessed in a shallow cavity, where separated flow conditions are present, could provide a solution for this problem. In order to reduce flow disturbances by the cavity and to obtain spatially resolved pressure data, a minimum sensor size has to be aimed for. The minimum diaphragm size, which meets the requirements for the measurement performance and sustains the higher mechanical stress due to the rough surface topography, has to be evaluated in an appropriate survey. Numerical simulations show that a sensor with a diaphragm size of $1 \mathrm{~mm}$ and an average pressure sensitivity of $4.5 \mathrm{~Hz} / \mathrm{Pa}$ is feasible, if the diaphragm thickness is reduced to $5 \mu \mathrm{m}$ and the maximum operating frequency is increased to $250 \mathrm{kHz}$ using a custom made CUT (Prochazka 2011). The roughened diaphragm top surface permits the optical interrogation of the resonator without the need for special alignment between sensor and detector. For sufficiently high Q-factors $(>50)$, the interrogation of the sensor using the SPDA and acoustic noise excitation shows a very good agreement with the reference method (laser vibrometer and optical excitation). Using a systematic frequency sweep during the "lock-in" detection, the targeted temporal resolution of $1 \mathrm{~Hz}$ cannot be achieved yet. As a faster alternative, pulse pair statistics can be used to process the demodulated image data of the SPDA (Miller et al. 1972; Meier et al. 2009). This demodulation approach is being tested with two SPDAs operating in parallel with the same demodulation frequency. The technique is attractive, as it can detect and analyze a complete range of frequencies without the need for a time-consuming frequency sweep. Despite the need for averaging over several frames, a temporal resolution of $1 \mathrm{~Hz}$ appears feasible considering the $5 \mathrm{kHz}$ frame rate of the SPDA.

\section{References}

Airaghi S (2006) Self-illuminating pressue sensitive paints using electrolumininescent foils, PhD Thesis ETH No. 16522, Swiss Federal Institute of Technology (ETHZ), Zurich, Switzerland

Beer S, Seitz P (2005) Real-time tomographic imaging without X-rays: a smart pixel array with massively parallel signal processing for realtime optical coherence tomography performing close to the physical limits. Res Microelectron Electron PhD 2:135-138 
Beltman WM, Van der Hoogt PJM, Spiering RMEJ, Tijdeman H (1998) Implementation and experimental validation of a new viscothermal acoustic finite element for Acousto-Elastic problems. J Sound Vib 216(1):159-185

Bennet JM, Mattsson L (1989) Introduction to surface roughness and scattering. Opt Soc Am. ISBN 1-55752-108-5

Blevins DB (2001) Formulas for natural frequency and mode shape. Krieger, NY

Bradshaw P, Goodman DG (1968) The effect of turbulence on staticpressure tubes. ARC R\&M 3527

Crocker JM (1998) Handbook of acoustics. Wiley, New York

Ecoffey S, Bouvet D, Ionescu AM, Fazan P (2002) Low-pressure chemical vapour deposition of nanograin polysilicon ultra-thin films. Nanotechnology 13(3):290-293

Fabula T, Buettgenbach S (1997) Resonant sensors: analytical and finite-element modeling of resonant silicon microsensors. Sens Mater 9(8):501-519

Fahy F, Gardonio P (2007) Sound and structural vibration: radiation, transmission and response. Elsevier/ Academic, 2007. ISBN 978-0-12-373633-8

Hayes M (1996) Statistical digital signal processing and modeling. Wiley, New York

Henning AK, Patel S, Selser M, Cozad BA (2004) Factors affecting silicon membrane burst strength. Proceedings of SPIE Vol. 5343:145-153

Lalanne P, Pichon P, Chavel P, Cambril E, Launois H (1999) Interferometric characterization of subwavelength lamellar gratings. Appl Opt 38(23):4980-4984

Lammerink TSJ, Elwenspoek M, Fluitman JHJ (1991) Optical excitation of micro-mechanical resonators. In: IEEE micro electro mechanical systems, MEMS, Nara, Japan, 1991, 'An Investigation of Micro Structures, Sensors, Actuators, Machines and Robots'

Lemmen RLC, Panuszka RJ (1996) Numerical evaluation of acoustic power radiation and radiation efficiencies of baffled plates. In: $\mathrm{V}$ School energy methods in vibroacoustics. Technical University of Mining and Metallurgy, Cracow, Poland
Liu T, Sullivan JP (2005) Pressure and temperature sensitive paints. Springer, Berlin

Manthey W, Kroemer N, Magori V (1992) Ultrasonic transducers and transducer arrays for applications in air. Meas Sci Technol 3:249-261

Meier AH, Roesgen T (2009) Heterodyne Doppler global velocimetry. Exp Fluids 47:665-672

Miller KS, Rochwarger MM (1972) A covariance approach to spectral moment estimation. IEEE Trans Info Theory 18:588-596

Pompeo LP (1992) An experimental study of three-dimensional turbulent boundary layers. PhD Thesis ETH No. 9780, Swiss Federal Institute of Technology (ETHZ), Zurich, Switzerland

Prochazka L (2011) Optically interrogated MEMS pressure sensor array, PhD Thesis ETH No. 19581, Swiss Federal Institute of Technology (ETHZ), Zurich, Switzerland

Rafiq M, Wykes C (1991) The performance of capacitive ultrasonic transducers using v-grooved backplates. Meas Sci Technol 2:168-174

Schlichting H, Gersten K, Krause E (2000) Boundary-layer theory. Springer, Berlin

Seifert A, Greenblatt D, Wygnanski IJ (2004) Active separation control: an overview of Reynolds and Mach numbers effects. Aerosp Sci Technol 8:569-582

Shaw R (1960) Influence of hole dimensions on static pressure measurements. J Fluid Mech 7:550-564

Stemme G (1991) Resonant silicon sensors. J Micromech Microeng $1: 113-125$

Sugiyama S, Baltes HP, Reed ML (1997) Resonant microsensors (special issue). Sens Mater 9(8):457-539

Tudor MJ, Beeby SP (1997) Resonant sensors: fundamentals and state of the art. Sens Mater 9(8):457-471

Uttamchandani D, Thornton KEB, Nixon J, Culshaw B (1987) Optically excited resonant diaphragm pressure sensor. El Lett 23(4):152-153

Zaman KBMQ, Bar-Sever A, Mangalam SM (1987) Effect of acoustic excitation on the flow over a low-Re airfoil. J Fluid Mech 182:127-148 